PROCEEDINGS OF THE

AMERICAN MATHEMATICAL SOCIETY

Volume 133, Number 7, Pages 2101-2107

S 0002-9939(05)07795-6

Article electronically published on January 31, 2005

\title{
SPACES OF TYPE BLO FOR NON-DOUBLING MEASURES
}

\author{
YINSHENG JIANG
}

(Communicated by Andreas Seeger)

\begin{abstract}
The spaces of type BLO for the positive Radon measures satisfying a growth condition on $\mathbb{R}^{d}$ are introduced. It is shown that some properties which hold for the classical space BLO when $\mu$ is a doubling measure remain valid for the spaces of type BLO introduced in this paper, without assuming $\mu$ doubling.
\end{abstract}

Let $d, n$ be some fixed integers with $d \geq 2,1 \leq n \leq d$, and let $\mu$ be a positive Radon measure on $\mathbb{R}^{d}$ satisfying the growth condition

$$
\mu(B(x, r)) \leq C_{0} r^{n} \quad \text { for all } x \in \mathbb{R}^{d}, r>0 .
$$

We do not assume that $\mu$ is doubling.

A kernel $k(\cdot, \cdot) \in L_{\text {loc }}^{1}\left(\mathbb{R}^{d} \times \mathbb{R}^{d} \backslash\{(x, y): x=y\}\right)$ is called a Calderón-Zygmund kernel if

1. $|k(x, y)| \leq \frac{C}{|x-y|^{n}}$,

2. there exists $0<\delta \leq 1$ such that

$$
\left|k(x, y)-k\left(x^{\prime}, y\right)\right|+\left|k(y, x)-k\left(y, x^{\prime}\right)\right| \leq C \frac{\left|x-x^{\prime}\right|^{\delta}}{|x-y|^{n+\delta}} \quad \text { if } \quad\left|x-x^{\prime}\right| \leq|x-y| / 2 .
$$

The Calderón-Zygmund operator associated to the kernel $k$ and the measure $\mu$ is formally defined as

$$
T f(x)=\int_{\mathbb{R}^{d}} k(x, y) f(y) d \mu(y) .
$$

We say that $T$ is bounded on $L^{p}(\mu)$ if the truncated operators

$$
T_{\epsilon} f(x)=\int_{|x-y|>\epsilon} k(x, y) f(y) d \mu(y)
$$

are bounded on $L^{p}(\mu)$ uniformly on $\epsilon>0$.

The maximal operator $T_{*}$ associated with the Calderón-Zygmund operator $T$ is defined as

$$
T_{*} f(x)=\sup _{\epsilon>0}\left|T_{\epsilon} f(x)\right|
$$

for $f \in L^{p}(\mu), p \in[1, \infty)$.

Received by the editors November 22, 2003 and, in revised form, March 24, 2004.

2000 Mathematics Subject Classification. Primary 42B20, 42B25, $42 \mathrm{~B} 35$.

Key words and phrases. Non-doubling measure, $\operatorname{RBLO}(\mu), \operatorname{RBMO}(\mu)$, Calderón-Zygmund maximal operator, maximal operator.

The author was supported in part by NSFC Grant \#10261007.

(C)2005 American Mathematical Society Reverts to public domain 28 years from publication 
When $\mu$ is a positive Radon measure satisfying the growth condition (1) and $T$ is bounded on $L^{2}(\mu)$, the weak type $(1,1)$ and $L^{p}(\mu)$ estimates for $T_{*}$ were obtained by F.Nazarov, S.Treil and A.Volberg in [4].

It is well known that if $\mu$ is Lebesgue measure and $T$ is bounded on $L^{2}\left(\mathbb{R}^{d}\right)$, then $T_{*}$ is bounded from $L^{\infty}\left(\mathbb{R}^{d}\right)$ into $B M O\left(\mathbb{R}^{d}\right)[5]$, and furthermore, is bounded from $L^{\infty}\left(\mathbb{R}^{d}\right)$ into $B L O\left(\mathbb{R}^{d}\right)$ [3]. For the positive Radon measure $\mu$ satisfying (1), $\mathrm{X}$.Tolsa 6] has introduced the spaces $R B M O(\mu)$, which are the suitable substitutes for the classical spaces $B M O\left(\mathbb{R}^{d}\right)$, and proved that if $T$ is bounded on $L^{2}(\mu)$, then $T$ is bounded from $L^{\infty}(\mu)$ into $R B M O(\mu)$.

In this note, for the positive Radon measure $\mu$ satisfying (1) we will introduce the spaces $R B L O(\mu)$ as the substitutes for the classical spaces $B L O\left(\mathbb{R}^{d}\right)$ defined by R.R.Coifman and R.Rochberg [2], and will show that if $T$ is bounded on $L^{2}(\mu)$, then $T_{*}$ is bounded from $L^{\infty}(\mu)$ into $R B L O(\mu)$.

We take notation and definitions from [6]. By a cube $Q \subset \mathbb{R}^{d}$ we mean a closed cube centered at some point $z_{Q} \in \operatorname{supp}(\mu)$ with sides parallel to the axes. Let $\alpha>1$ and $\beta>\alpha^{n}$. A cube with side length $l(Q)$ is said to be $(\alpha, \beta)$-doubling if $\mu(\alpha Q) \leq \beta \mu(Q)$, where $\alpha Q$ denotes the cube concentric with $Q$ and having side length $\alpha l(Q)$. Given a cube $Q \subset \mathbb{R}^{d}$, let $N$ be the smallest integer $\geq 0$ such that $\alpha^{N} Q$ is doubling. Denote this doubling cube by $\widetilde{Q}$. If $\alpha$ and $\beta$ are not specified, by a doubling cube we will mean a $\left(4 \sqrt{d},(4 \sqrt{d})^{n+1}\right)$-doubling cube. Given two cubes $Q \subset R$, we set

$$
K_{Q, R}=1+\sum_{k=1}^{N_{Q, R}} \frac{\mu\left(2^{k} Q\right)}{l\left(2^{k} Q\right)^{n}}
$$

where $N_{Q, R}$ is the first integer $k$ such that $2^{k} Q \supset R$.

Let $\rho>1$ be some fixed constant. Say that $f \in L_{\text {loc }}^{1}(\mu)$ belongs to $R B M O(\mu)$ if there exists some constant $C_{1}$ such that for any cube $Q$,

$$
\frac{1}{\mu(\rho Q)} \int_{Q}\left|f-m_{\widetilde{Q}}(f)\right| d \mu \leq C_{1}
$$

and

$$
\left|m_{Q}(f)-m_{R}(f)\right| \leq C_{1} K_{Q, R} \quad \text { for any two doubling cubes } Q \subset R,
$$

where $m_{Q}(f)=\mu(Q)^{-1} \int_{Q} f d \mu$. The best constant $C_{1}$ is the $R B M O(\mu)$ norm of $f$, which we denote as $\|f\|_{*}$ and is independent of $\rho>1$. It is shown by X. Tolsa [ [6] that one can replace (3) by

$$
\frac{1}{\mu(Q)} \int_{Q}\left|f-m_{Q}(f)\right| d \mu \leq C_{2} \quad \text { for any doubling cube } Q,
$$

or by

(6) $\left(\frac{1}{\mu(\rho Q)} \int_{Q}\left|f-m_{\widetilde{Q}}(f)\right|^{p} d \mu\right)^{1 / p} \leq C_{3} \quad$ for $1 \leq p<\infty$ and any cube $Q$,

and the best constants $C_{2}$ and $C_{3}$ are comparable to $\|f\|_{*}$.

We are ready to define the spaces of type $B L O$ for the positive Radon measure $\mu$ satisfying (1). 
Definition 1. We say that $f \in L_{\text {loc }}^{1}(\mu)$ belongs to $R B L O(\mu)$ if there exists some constant $C_{4}$ such that for any doubling cube $Q$,

$$
m_{Q}(f)-\underset{x \in Q}{\operatorname{essinf}} f(x) \leq C_{4}
$$

and

$$
m_{Q}(f)-m_{R}(f) \leq C_{4} K_{Q, R} \quad \text { for any two doubling cubes } Q \subset R .
$$

The smallest constant $C_{4}$ will be denoted by $\|f\|_{R B L O}$.

Observe that (7) and (8) are equivalent to (7) and (4). It is easy to check that $L^{\infty}(\mu) \subset R B L O(\mu)$ with $\|f\|_{R B L O} \leq 2\|f\|_{L^{\infty}(\mu)}$ and $R B L O(\mu) \subset R B M O(\mu)$ with $\|f\|_{*} \leq 2\|f\|_{R B L O}$.

C.Bennett [1] has obtained a criterion for the classical spaces $B L O\left(\mathbb{R}^{d}\right)$. To give out the $R B L O(\mu)$ criterion we consider the non-centered doubling maximal function

$$
M f(x)=\sup _{\substack{Q \ni x \\ Q: \text { doubling }}} m_{Q}(f)=\sup _{\substack{Q \ni x \\ Q: \text { doubling }}} \frac{1}{\mu(Q)} \int_{Q} f d \mu,
$$

where the supremum extends over all doubling cubes $Q$ containing $x$. By the Lebesgue differentiation theorem, $M f(x) \geq f(x) \mu$-a.e. $x \in \mathbb{R}^{d}$. Moreover, $|M f(x)|$ $\leq M(|f|)(x):=N f(x)$. The maximal operator $N$ is weak type $(1,1)$ and bounded on $L^{p}(\mu)$ for $1<p \leq \infty$ [6] , so is $M$.

Lemma 1. $f \in R B L O(\mu)$ if and only if $M f-f \in L^{\infty}(\mu)$ and $f$ satisfies (8). Furthermore,

$$
\|M f-f\|_{L^{\infty}(\mu)} \sim\|f\|_{R B L O} .
$$

Proof. Suppose first that $f \in R B L O(\mu)$. Then $f$ satisfies (8). Observe that (see 6. Remark 2.3]), by the Lebesgue differentiation theorem, for $\mu$-a.e. $x \in \mathbb{R}^{d}$ one can find a sequence of doubling cubes $\left\{Q_{k}\right\}_{k}$ centered at $x$ with $l\left(Q_{k}\right) \rightarrow 0$ such that

$$
\lim _{k \rightarrow \infty} \frac{1}{\mu\left(Q_{k}\right)} \int_{Q_{k}} f d \mu=f(x) .
$$

Let $x$ be any such point, and let $Q$ be any doubling cube containing $x$. Then $f(x) \geq \underset{x \in Q}{\operatorname{essinf}} f(x)$ and so

$$
m_{Q}(f)-f(x) \leq m_{Q}(f)-\underset{x \in Q}{\operatorname{essinf}} f(x) \leq\|f\|_{R B L O} .
$$

Taking the supremum over all doubling cubes containing $x$, we get

$$
M f(x)-f(x) \leq\|f\|_{R B L O} .
$$

Hence $M f-f \in L^{\infty}(\mu)$ and $\|M f-f\|_{L^{\infty}(\mu)} \leq\|f\|_{R B L O}$.

Conversely, suppose $M f-f \in L^{\infty}(\mu)$ and let $Q$ be any doubling cube in $\mathbb{R}^{d}$. If any $x \in Q$ is such that

$$
f(x)<m_{Q}(f)-\|M f-f\|_{L^{\infty}(\mu)},
$$

then

$$
M f(x)-f(x) \geq m_{Q}(f)-f(x)>\|M f-f\|_{L^{\infty}(\mu)} .
$$

So, for $\mu$-a.e. $x \in Q$,

$$
f(x) \geq m_{Q}(f)-\|M f-f\|_{L^{\infty}(\mu)},
$$


and consequently,

$$
\underset{x \in Q}{\operatorname{essinf}} f(x) \geq m_{Q}(f)-\|M f-f\|_{L^{\infty}(\mu)} .
$$

Since $f$ satisfies (8) we get that $f \in R B L O(\mu)$ and

$$
\|f\|_{R B L O} \leq C\|M f-f\|_{L^{\infty}(\mu)} .
$$

Our main results are as follows.

Theorem 1. If the Calderón-Zygmund operator $T$ is bounded on $L^{2}(\mu)$, then the maximal operator $T_{*}$ is bounded from $L^{\infty}(\mu)$ into $R B L O(\mu)$.

Theorem 2. If $f \in R B M O(\mu)$ and $M f$ satisfies (8), then $M f \in R B L O(\mu)$ and

$$
\|M f\|_{R B L O} \leq C\|f\|_{*} .
$$

In particular, $M$ is bounded on $R B L O(\mu)$.

Theorem 3. A locally integrable function $f$ belongs to $R B L O(\mu)$ if and only if there exist $h \in L^{\infty}(\mu)$ and $F \in R B M O(\mu)$ with $M F$ satisfying (8) such that

$$
f=M F+h .
$$

Furthermore,

$$
\|f\|_{R B L O} \sim \inf \left(\|F\|_{*}+\|h\|_{L^{\infty}(\mu)}\right)
$$

where the infimum extends over all representations of the form (11).

The proof of Theorem 1. Let $x \in \mathbb{R}^{d} \cap \operatorname{supp}(\mu)$ and $Q$ be any doubling cube containing $x$. For each fixed cube $Q$ let $B$ be the smallest ball centered at $x$ which contains $Q$. Then $2 B \subset 4 \sqrt{d} Q$. If $f \in L^{\infty}(\mu) \cap L^{p_{0}}(\mu)$ for some $p_{0} \in[1, \infty)$, by $L^{2}(\mu)$ boundedness of $T_{*}$, 4, we have

$$
\begin{aligned}
\frac{1}{\mu(Q)} \int_{Q} T_{*}\left(f \chi_{2 B}\right) d \mu & \leq \frac{1}{\mu(Q)^{1 / 2}}\left\{\int_{\mathbb{R}^{d}}\left[T_{*}\left(f \chi_{2 B}\right)\right]^{2} d \mu\right\}^{1 / 2} \\
& \leq \frac{C}{\mu(Q)^{1 / 2}}\left\{\int_{\mathbb{R}^{d}}\left|f \chi_{2 B}\right|^{2} d \mu\right\}^{1 / 2} \\
& \leq C \frac{\mu(2 B)^{1 / 2}}{\mu(Q)^{1 / 2}}\|f\|_{L^{\infty}(\mu)} \\
& \leq C \frac{\mu(4 \sqrt{d} Q)^{1 / 2}}{\mu(Q)^{1 / 2}}\|f\|_{L^{\infty}(\mu)} \\
& \leq C\|f\|_{L^{\infty}(\mu)}
\end{aligned}
$$

From (2) it follows that

$$
T_{*}\left(f \chi_{\mathbb{R}^{d} \backslash 2 B}\right)(x) \leq T_{*} f(x) .
$$

By this and the conditions of the Calderón-Zygmund kernel, for all $y \in Q$, we have

$$
\begin{aligned}
T_{*}\left(f \chi_{\mathbb{R}^{d} \backslash 2 B}\right)(y) & \leq\left|T_{*}\left(f \chi_{\mathbb{R}^{d} \backslash 2 B}\right)(y)-T_{*}\left(f \chi_{\mathbb{R}^{d} \backslash 2 B}\right)(x)\right|+T_{*}\left(f \chi_{\mathbb{R}^{d} \backslash 2 B}\right)(x) \\
& \leq C\|f\|_{L^{\infty}(\mu)}+T_{*} f(x) .
\end{aligned}
$$

Therefore,

$$
\frac{1}{\mu(Q)} \int_{Q} T_{*}\left(f \chi_{\mathbb{R}^{d} \backslash 2 B}\right) d \mu \leq C\|f\|_{L^{\infty}(\mu)}+T_{*} f(x) .
$$


From this and (13), we get

$$
\frac{1}{\mu(Q)} \int_{Q} T_{*} f d \mu \leq C\|f\|_{L^{\infty}(\mu)}+T_{*} f(x) .
$$

So,

$$
\left\|M\left(T_{*} f\right)-T_{*} f\right\|_{L^{\infty}(\mu)} \leq C\|f\|_{L^{\infty}(\mu)} .
$$

From this and Lemma 1, it suffices to show that $T_{*} f$ satisfies (8). We apply the argument analogous to [6, pp. 104-105]. Let $Q \subset R$ be any two doubling cubes. Recall that $N_{Q, R}$ is the first integer $k$ such that $2^{k} Q \supset R$. We denote $Q_{R}=$ $2^{N_{Q, R}+1} Q$. Thus, for $x \in Q$ and $y \in R$, we set

$$
\begin{aligned}
T_{\epsilon} f(x)= & T_{\epsilon}\left(f \chi_{2 Q}\right)(x)+\sum_{k=1}^{N_{Q, R}} T_{\epsilon}\left(f \chi_{2^{k+1} Q \backslash 2^{k} Q}\right)(x)+T_{\epsilon}\left(f \chi_{\mathbb{R}^{d} \backslash Q_{R}}\right)(x) \\
& -\left(T_{\epsilon}\left(f \chi_{Q_{R}}\right)(y)+T_{\epsilon}\left(f \chi_{\mathbb{R}^{d} \backslash Q_{R}}\right)(y)\right)+T_{\epsilon} f(y) .
\end{aligned}
$$

Since

$$
\left|T_{\epsilon}\left(f \chi_{\mathbb{R}^{d} \backslash Q_{R}}\right)(x)-T_{\epsilon}\left(f \chi_{\mathbb{R}^{d} \backslash Q_{R}}\right)(y)\right| \leq C\|f\|_{L^{\infty}(\mu)},
$$

we get

$$
\begin{aligned}
T_{*} f(x) \leq & T_{*}\left(f \chi_{2 Q}\right)(x)+C\left(1+\sum_{k=1}^{N_{Q, R}} \frac{\mu\left(2^{k+1} Q\right)}{l\left(2^{k+1} Q\right)^{n}}\right)\|f\|_{L^{\infty}(\mu)} \\
& +T_{*}\left(f \chi_{Q_{R}}\right)(y)+T_{*} f(y) .
\end{aligned}
$$

Now we take the mean over $Q$ for $x$, and over $R$ for $y$. We write

$$
m_{R}\left(T_{*}\left(f \chi_{Q_{R}}\right)\right) \leq m_{R}\left(T_{*}\left(f \chi_{Q_{R} \cap 2 R}\right)\right)+m_{R}\left(T_{*}\left(f \chi_{Q_{R} \backslash 2 R}\right)\right) .
$$

Similar to the previous estimate (13) we obtain

$$
m_{Q}\left(T_{*}\left(f \chi_{2 Q}\right)\right) \leq C\|f\|_{L^{\infty}(\mu)}
$$

and

$$
m_{R}\left(T_{*}\left(f \chi_{Q_{R} \cap 2 R}\right)\right) \leq C\|f\|_{L^{\infty}(\mu)} .
$$

On the other hand, since $l\left(Q_{R}\right) \approx l(R)$, we have

$$
m_{R}\left(T_{*}\left(f \chi_{Q_{R} \backslash 2 R}\right)\right) \leq C\|f\|_{L^{\infty}(\mu)} .
$$

Therefore,

$$
m_{Q}\left(T_{*} f\right)-m_{R}\left(T_{*} f\right) \leq C K_{Q, R}\|f\|_{L^{\infty}(\mu)} .
$$

If $f \notin L^{p}(\mu)$ for all $p \in[1, \infty)$, then the integral $\int_{|x-y|>\epsilon} k(x, y) f(y) d \mu(y)$ may not be convergent. The operator $T_{\epsilon}$ can be extended to the whole space $L^{\infty}(\mu)$ following the standard arguments: Given a cube $Q_{0}$ centered at the origin with side length $l\left(Q_{0}\right)>3 \epsilon$, we write $f=f_{1}+f_{2}$, with $f_{1}=f \chi_{2 Q_{0}}$. For $x \in Q_{0}$, we define

$$
T_{\epsilon} f(x)=T_{\epsilon} f_{1}(x)+\int_{|x-y|>\epsilon}(k(x, y)-k(0, y)) f_{2}(y) d \mu(y) .
$$

Now both integrals in this equation are convergent. With arguments similar to the case $f \in L^{\infty}(\mu) \cap L^{p_{0}}(\mu)$ we complete the proof. 
To prove Theorem 2 and Theorem 3 we require the following lemma.

Lemma 2. If $f \in R B M O(\mu)$, then for any doubling cube $Q$,

$$
\frac{1}{\mu(Q)} \int_{Q} M f d \mu \leq C\|f\|_{*}+\underset{x \in Q}{\operatorname{sssinf}} M f(x) .
$$

Moreover, if $M f$ is finite $\mu$-a.e., then

$$
\frac{1}{\mu(Q)} \int_{Q} M f d \mu-\underset{x \in Q}{\operatorname{essinf}} M f(x) \leq C\|f\|_{*}
$$

Proof. Set $\rho=4 \sqrt{d} / 5$ in (3). Fix a doubling cube $Q$. For $f \in R B M O(\mu)$ we write

$$
f=\left(f-m_{Q}(f)\right) \chi_{3 Q}+\left(m_{Q}(f) \chi_{3 Q}+f \chi_{\mathbb{R}^{d} \backslash 3 Q}\right) .
$$

Since $M$ is bounded on $L^{2}(\mu)$, we have

$$
\begin{aligned}
& \int_{Q} M\left(\left(f-m_{Q}(f)\right) \chi_{3 Q}\right) d \mu \\
\leq & \mu(Q)^{1 / 2}\left\{\int_{\mathbb{R}^{d}}\left|M\left(\left(f-m_{Q}(f)\right) \chi_{3 Q}\right)\right|^{2} d \mu\right\}^{1 / 2} \\
\leq & C \mu(Q)^{1 / 2}\left\{\int_{\mathbb{R}^{d}}\left|\left(f-m_{Q}(f)\right) \chi_{3 Q}\right|^{2} d \mu\right\}^{1 / 2} \\
\leq & C \mu(Q)^{1 / 2}\left(\left\{\int_{3 Q}\left|f-m_{\widetilde{3 Q}}(f)\right|^{2} d \mu\right\}^{1 / 2}+\left\{\int_{3 Q}\left|m_{Q}(f)-m_{\widetilde{3 Q}}(f)\right|^{2} d \mu\right\}^{1 / 2}\right) \\
\leq & C \mu(Q)^{1 / 2}\left(\mu((12 \sqrt{d} / 5) Q)^{1 / 2}\|f\|_{*}+\mu(3 Q)^{1 / 2}\|f\|_{*} K_{Q, \widetilde{3 Q}}\right) \\
\leq & C \mu(Q)^{1 / 2} \mu(4 \sqrt{d} Q)^{1 / 2}\|f\|_{*} \leq C \mu(Q)\|f\|_{*} .
\end{aligned}
$$

Next, we shall show that

$$
\frac{1}{\mu(Q)} \int_{Q} M\left(m_{Q}(f) \chi_{3 Q}+f \chi_{\mathbb{R}^{d} \backslash 3 Q}\right) d \mu \leq C\|f\|_{*}+\underset{x \in Q}{\operatorname{essinf}} M f(x) .
$$

It suffices to show that

$$
M\left(m_{Q}(f) \chi_{3 Q}+f \chi_{\mathbb{R}^{d} \backslash 3 Q}\right)(x) \leq C\|f\|_{*}+\underset{x \in Q}{\operatorname{essinf}} M f(x) \quad \mu \text {-a.e. } x \in Q .
$$

For this it will be enough to show that

$$
\frac{1}{\mu(R)} \int_{R}\left(m_{Q}(f) \chi_{3 Q}+f \chi_{\mathbb{R}^{d} \backslash 3 Q}\right) d \mu \leq C\|f\|_{*}+\underset{x \in Q}{\operatorname{essinf}} M f(x)
$$

for any doubling cube $R \ni x$. If $R \subset 3 Q$, the result follows immediately:

$$
\frac{1}{\mu(R)} \int_{R}\left(m_{Q}(f) \chi_{3 Q}+f \chi_{\mathbb{R}^{d} \backslash 3 Q}\right) d \mu=m_{Q}(f) \leq \underset{x \in Q}{\operatorname{essinf}} M f(x) .
$$

So, suppose $R \cap\left(\mathbb{R}^{d} \backslash 3 Q\right) \neq \emptyset$. Then $l(R)>l(Q)$ and $3 Q \subset 5 R$. Write $m_{Q}(f) \chi_{3 Q}+f \chi_{\mathbb{R}^{d} \backslash 3 Q}=\left(m_{Q}(f)-m_{\widetilde{5 R}}(f)\right) \chi_{3 Q}+\left(f-m_{\widetilde{5 R}}(f)\right) \chi_{\mathbb{R}^{d} \backslash 3 Q}+m_{\widetilde{5 R}}(f)$. 
Obviously, $m_{\widetilde{5 R}}(f) \leq \underset{x \in Q}{\operatorname{essinf}} M f(x)$. Further,

$$
\begin{aligned}
& \int_{R}\left\{\left(m_{Q}(f)-m_{\widetilde{5 R}}(f)\right) \chi_{3 Q}+\left(f-m_{\widetilde{5 R}}(f)\right) \chi_{\mathbb{R}^{d} \backslash 3 Q}\right\} d \mu \\
\leq & \mu(3 Q)\left|m_{Q}(f)-m_{\widetilde{5 R}}(f)\right|+\int_{5 R}\left|f-m_{\widetilde{5 R}}(f)\right| \chi_{\mathbb{R}^{d} \backslash 3 Q} d \mu \\
\leq & \frac{\mu(4 \sqrt{d} Q)}{\mu(Q)} \int_{Q}\left|f-m_{\widetilde{5 R}}(f)\right|+\int_{5 R \backslash 3 Q}\left|f-m_{\widetilde{5 R}}(f)\right| d \mu \\
\leq & C \int_{5 R}\left|f-m_{\widetilde{5 R}}(f)\right| d \mu \\
\leq & C \mu(4 \sqrt{d} R)\|f\|_{*} \leq C \mu(R)\|f\|_{*} .
\end{aligned}
$$

This establishes (18) and completes the proof of Lemma 2

The proof of Theorem 2. By Lemma 2, it suffices to prove that if $f \in R B L O(\mu)$, then $M f$ satisfies (8). In fact, applying Lemma 1 for any two doubling cubes $Q \subset R$ we have

$$
\begin{aligned}
& m_{Q}(M f)-m_{R}(M f) \\
\leq & m_{Q}(M f)-m_{Q}(f)+m_{R}(M f)-m_{R}(f)+\left|m_{Q}(f)-m_{R}(f)\right| \\
\leq & 2\|M f-f\|_{L^{\infty}(\mu)}+\|f\|_{*} K_{Q, R} \\
\leq & 2\|f\|_{R B L O}+\|f\|_{*} K_{Q, R} \\
\leq & C\|f\|_{R B L O} K_{Q, R} .
\end{aligned}
$$

As we remarked above, this completes the proof of Theorem 2

Theorem 3 follows immediately from Lemma 1 and Theorem 2, and we omit the details here.

\section{ACKNOWLEDGEMENT}

I would like to thank the referee for the helpful comment which improved the presentation of this paper.

\section{REFERENCES}

1. C. Bennett, Another characterization of BLO, Proc. Amer. Math. Soc., 85(1982), 552-556. MR0660603 (84h:42029)

2. R. R. Coifman and R. Rochberg, Another characterization of BMO, Proc. Amer. Math. Soc., 79(1980), 249-254. MR 0565349 (81b:42067)

3. M. A. Leckband, Structure results on the maximal Hilbert transform and two-weight norm inequalities, Indiana Univ. Math. J., 34 (1985), 259-275. MR0783915 (86k:42016)

4. F. Nazarov, S. Treil and A. Volberg, Weak type estimates and Cotlar's inequalities for Calderón-Zygmund operators on non-homogeneous spaces, Internat. Math. Res. Notices, 9 (1998), 463-487. MR.1626935 (99f:42035)

5. E. M. Stein, Singular integral, harmonic functions, and differentiability properties of functions of several variables, Proc. Sympos. Pure Math., vol. 10, Amer. Math. Soc., 1967, 316-335. MR0482394 (58:2467)

6. X. Tolsa, BMO, $H^{\perp}$ and Calderón-Zygmund operators for non doubling measures, Math. Ann., 319 (2001), 89-149. MR.1812821 (2002c:42029)

Department of Mathematics, Xinjiang University, Urumgi, 830046, People's Republic OF China

E-mail address: ysjiang@xju.edu.cn 